\title{
Dans le sillage de Maurice Gariel...
}

\section{La grande famille des turbines et des pompes

\author{
The vast turbine and pump family
}

\author{
par PAUL BERGERON
}

PRESIDENT DIRECTEUR GÉNERAI, DE LA MAISON L. BERGEION, PARIS

\begin{abstract}
Les theories de base des turbines et des pompes peubent etre menées naralletement.

Cependant, les transformations d'energie ans les deux types de machine sont inversées, ce ghi se traduit pur des observations en apparence sans liens directs et pratiquement par des proportions trés éloignées les unes des autres pour les mémes vitesses spécifiques. Ceci a pu faire croire a des techniques differentes.

Les realisations relativement récentes des aurbines-pompes réversibles rendent anjourdhui évidente la parenté étroite entre turbines el pompes et l'intérét que pent présenter la confrontation des expériences des spécialistes qui ont observé des machines analogues de points de vile diamitralement opposés.

Cette conclusion permet de donner toute sa valenr a la collaboration intime aue nous avons eue depuis plus de irente années avec M. M. Gariel et son équipe.
\end{abstract}

Pendant plus de trente années, j'ai été de ceux qui ont eu le grand privilege d'approcher de suf. fisamment près M. Gariel pour dépasser de beaucoup le cadre banal des relations d'affaires.

Bien des personnes ont pris avant moi la parole ou la plume pour exprimer qui était M. Gariel et l'exprimer en des termes plus éloguents que je ne saurais faire. Pour moi, je dirai simplement que j'ai été dès l'origine tout naturellement et irrésistiblement attiré par sa valeur. morale et spirituelle, subjugué par sa finesse et son érudition, éprouvant pour lui sans interruption, et quelles que soient les situations, des sentiments d'admiration et de profonde sympathie.

J'irai plus loin en disant qu'on ne peut parler de Maurice Gariel sans faire intervenir les sentiments, mème dans la technique. Veuillez donc accepter que les deux s'entremêlent ici, d'autant plus que ie ne puis dissocier dans mon esprit le souvenir de M. Gariel et celui de mon Père.

Pendant plus de vingt années, j'ai suivi de près ou de loin les discussions passionnantes ou même passionnées qui ont pu avoir lieu entre ces deux

\begin{abstract}
Though basic theoretical research on both turbines and pumps can run along similar lines, the tuvo tijpes of machine nevertheless involve. cractly opposite energy conversion processes. This results in apparently unrelated observattions and widely differing machine dimensions for the same specific speed, thus giving the impression that two different techniques are concerned.

However, the comparatively recent development of reversible pump turbines has shown hou closelly related pumps and turbines really are and the advantages that can be gained by comparing the experience of specialists who have observed similar machines from diametricall! opposite points of view.

This conclusion clearly expresses the full value of our thirty years of close collaboration with M. Gariel and his research team.
\end{abstract}

êtres exceptionnels sur tous les sujets, religieux, artistiques, humains ou techniques.

Que ce soit sur le plan spirituel ou sur le plan technique, les conditions étaient cependant très particulières :

Tous les deux étaient profondément chrétiens, tous les deux étaient hydrauliciens, mais tandis que l'un était catholique, l'autre était protestant; tandis que l'un était plus particulièrement orienté vers les turbines, l'autre l'était vers les pompes, c'est-à-dire que tous les deux étaient de la même famille spirituelle et intellectuelle, mais ils étaient d'églises et de chapelles différentes.

Pour des esprits d'élite, ces points de vue différents sur des sujets analogues, loin d'apporter l'incompréhension ou même la discorde commc bien souvent, ne pouvaient conduire gu'à des ćchanges féconds et à des prises de position saines et créatrices.

Devant un tel exemple et sous de tels auspices, i'ai tout naturellement continué à croire aussi à l'efficacité de ces rapports et même à une collaboration plus intime sur le plan technique. 


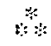

Puisqu'il m'est donné de parler aujourd'hui des échanges d'expériences entre turbiniers et pompiers, je profite de l'oceasion pour dévier la question sur le plan général et essayer d'exposer ce qu'il peut y avoir de commun ou de dissemblable entre les deux types de machines, l'origine des incompréhensions réciproques initiales, l'état actuel du problème, en un mot de faire un historique rapide de la question.

Comme l'exposé déhute obligatoirement par des considérations techniques, il est nécessaire (et je m'en excuse auprès des initiés) de faire un rappel succinct des théories de base, ne serait-ce que pour bien définir les termes utilisés dans la suite. En effet, nous utilisons Jes uns et les autres souvent un jargon différent et ne parlons pas toujours la mème langue, ce qui est ecrtainement à l'origine d'une difficulté d'échanger des idées et même de se comprendre.

Il y a lieu de remarquer tout d'abord que pour toutes les propriétés qui résultent de l'application pure et simple du théorème d'Euler, les théories peuvent être menées parallèlement. C'est d'ailleurs la présentation qu'a toujours adoptée Louis Bergeron dans ses ouvages et dans ses cours, ot ceci sous une forme entierement personnelle et originale parfaitement mise au point jusqu'à devenir classique, spécialement en France, ne dissociant les deux types de machines qu'après un exposé général unique.

Je vais en résumer très brièvement les conclusions :

- Soit une roue type Francis et un triangle des vitesses au grand diamètre $A B C$ pour la pompe et le même triangle inversé $A B^{\prime} C^{\prime}$ pour la turbine (fig. 1), ces triangles correspondant au point de fonctionnement optimal, c'està-dire au point pour lequel la machine a été dimensionnée.

En supposant d'abord le moment de vilesse nul au petit diametre, le théorime d'Euler donne:

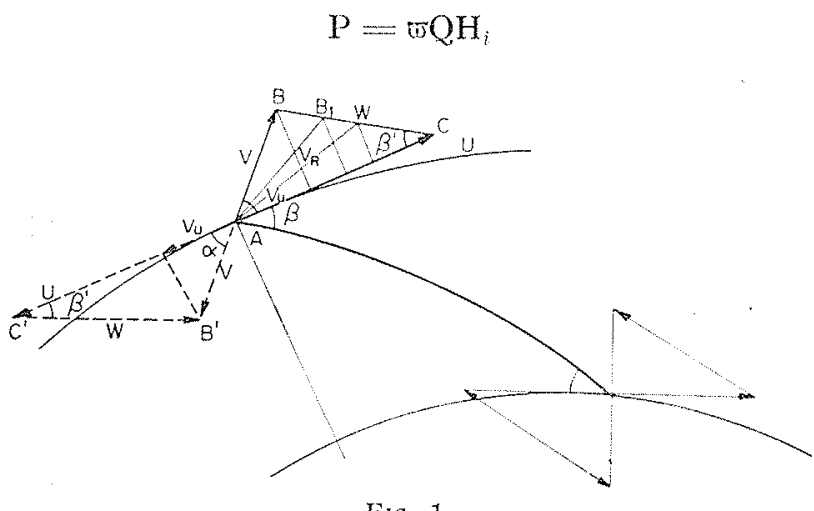

où $P$ est la puissance sur l'arbre compte non tenu des pertes mécaniques, $Q$ le débit et :

$$
\mathrm{H}_{i}=\left[\frac{\mathrm{UVu}}{g}\right]_{\mathrm{D}}
$$

Il y a lieu de noter que $\mathrm{H}_{i}$, qui est une hauteur, se déduit de $\mathrm{P}$ et est indépendant de toutes les pertes hydrauliques.

Dans la représentation à vitesse constante avec $\mathrm{H}$ en ordonnée et $\mathrm{Q}$ en abscisse, le point représentatif $M$ pour pompe et turbine est le mème, puisque les triangles ont été pris identiques et correspondent en principe aux angles pour lesquels la machine a été calculée (fig. 2).

Fin restant toujours dans la représentation à vitesse constante lorsque le débit varie :

1. Pour la pompe, si l'on suppose en première approximation que l'angle $\beta$ ', qui est lié à $\beta$ angle

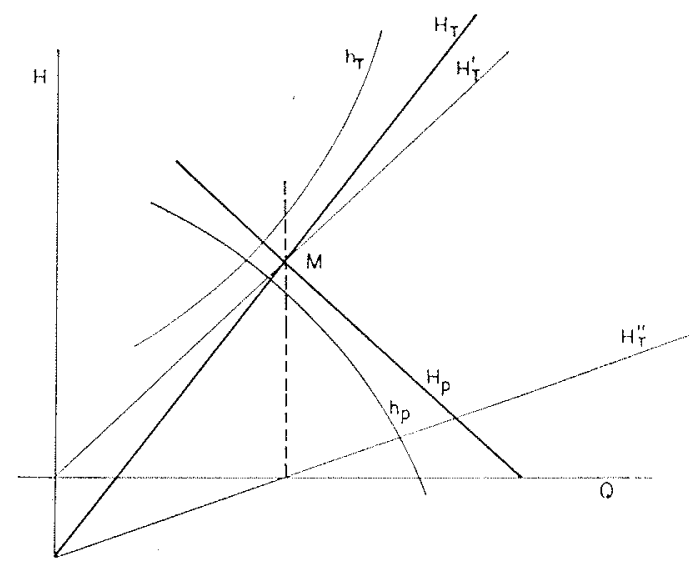

F10. 2

de l'aube, reste constant, le triangle se déforme de telle manière que $U$ reste constant ainsi que $\beta^{\prime}$, tandis que $\alpha$ varic ( $V_{\mathrm{r}}$ est proportionnel au débit). Dans ce cas, la caractéristique $\mathrm{H}_{i}$ en fonction de $Q$ est une droite $H_{1}$, de coefficient angulaire négatif, fonction lui-mème de l'angle? qu'impose le moment de vitesse au grand diamètre,

2" Pour la turbine, c'est l'angle a du distributeur qui reste constant et impose le moment de vitesse $U$. On trouve alors que la caractéristique de $\mathrm{H}_{i}$ est une droite $\mathrm{H}^{\prime}{ }_{\mathrm{T}}$ de coefficient angulaire positif, fonction de $\alpha$.

D’après ce qui précède, les deux droites passent évidemment par le même point $M$, mais pour être dans les deux machines le fonctionnement optimal, il faudra, pour la pompe, que le diffuseur soit calculé pour l'angle $\alpha$ tandis que, pour la turbine, il faudra que $\beta^{\prime}$ de l'eau s'accommode au mieux de $\beta$ de l'aube.

Pour les pompes, sauf construction particulière, il faut considérer qu'il n'y a pas rotation au petit diamedre. Par contre, pour les turbi- 
nes, la théorie simplifiée montre aisément que l'angle de sortie de la vitesse relative étant imposé par l'angle de construction de l'aube, il y a rotation au petit diamètre qui doit au mieux s'annuler pour le point $\mathrm{M}$ pour lequel la machine est calculée, et d'autre part que le moment de vitesse $[\mathrm{UVu} / \mathrm{g}]$ à la sortie varie linéairement avec le débit, d'où la droite $\mathrm{H}^{\prime \prime}{ }_{\text {T. }}$. Comme le UVu au petit diamètre est à ajouter au UVu grand diamètre, on a finalement, en turbine, pour la caractéristique de $\mathrm{H}_{i}$, une droite $\mathrm{H}_{\mathrm{r}}$ obtenue en faisant la somme des ordonnées de $\mathrm{H}^{\prime}{ }_{\mathrm{T}}$ et $\mathrm{H}^{\prime \prime}{ }_{\mathrm{T}}$.

A cos droites, il faut ajouter les pertes pour la turbine, les retrancher pour la pompe, pertes d'allure parabolique avec minimum au voisinage du point $M$, ce qui donne respectivement ha courbe $h_{I^{\prime}}$ de chute nette el la courbe $h_{p}$ de hauteur recueillie.

La pratique confirme cette théorie en apparence sommaire, en ce sens qu'en partant des essais d'une machine, on peut calculer les courbes $\mathrm{H}$ (simplement d'après la courbe de la puissance en fonction de $q$ ) et qu'à vitesse constante on trouve bien une droite pour les pompes dans une zone étendue autour du fonctionnement normal, et une droite pratiquement rigoureuse pour les turbines. In raisonnement inverse permet de tirer des valeurs de $\mathrm{H}$ expérimentales le compor. tement interne de la machine comme précisé cidessous.

Jusque-là, les théories peuvent être menées parallèlement; les raisonnements sont symétriques, il suffit de changer le sens des écoulements.

Toutefois, une première remarque s'impose sur la manière différente dont ces résultats de base ont été utilisés. Si la droite $\mathrm{H}_{p}$ a été largement exploitée par les constructeurs de pompes, la droite $H_{\mathrm{T}}$ n'a pas attiré spécialement l'attention des constructeurs de turbines, tout au moins pendant longtemps, et ceci malgré I'insistance qu'avait mise L. Bergeron pour essayer d'en divulguer l'intérêt.

Les raisons, à notre avis, en sont les suivantes:

a) Une raison d'ordre pratique : la représentation à vitesse constante est habituelle en pompe, tandis que, pour les turbines, la représentation sous chute constante est normalement utilisée. Elle est en effet la seule pratique pour les turbines, mais ne permet pas de mettre en évidence la courbe des $H_{\mathrm{T}}$ et masque l'intérêt qu'elle peut présenter;

b) Une raison d'intérêt technique : capitale pour les pompes et d'intérêt moins impératif pour les turbines.

En effet, les courbes $H$ déduites de la courbe expérimentale de la puissance en fonction des débits, en donnant UVu en chaque point, permet de connaitre le triangle, c'est-à-dire aussi l'angle $\beta^{\prime}$ toujours très différent de l'angle $\beta$ des aubes, pour les pompes.

En relevant simultanément les différentes pressions moyennes entre le grand et le petit diamètre, on dispose de tous les éléments pour séparer les pertes entre roue et diffuseur. D'où une connaissance totale du fonctionnement de la machine sans aucun sondage.

Pour les turbines, la droite $\mathrm{H}_{\mathrm{T}}$ présente peutêtre un intérêt moins immédiat, car dans le triangle d'entrée, l'angle z imposé par les directrices étant à peu près conmu, le triangle au grand diamètre l'est aussi. Par contre, elle permet de connaître le moment de vitesse moyen au petit diamètre de la rone, ce qui n'est certes pas négligeable, mais d'un intérêt moins capital que la connaissance du triangle réel à la sortie d'une roue de pompe déformé par l'écart angulaire.

Elle permet aussi la séparation des pertes, à la condition de mesurer aussi la différence des pressions moyennes entre entrée et sortie de la roue, et ceci est d'un grand intérêt.

$$
* ;
$$

Comme déjà dit, jusqu'ici les théories ont pu être menées parallèlement. Mais les divergences profondes apparaissent quand, dépassant le cadre du théorème d'Euler, on aborde la question des pertes hydrauliques et des phénomènes qui les créent.

Ces différences peuvent se concrétiser sommairement en deux remarques principales:

a) Sur le plan théorique, et seulement dans le cas particulier des Francis et centrifuges, le champ centrifuge dans la roue s'oppose à l'écoulement dans les turbines et le facilite dans les pompes, d'où un comportement très différent des écoulements, surtout dans les fonctionnements s'écartant du régime optimal pour lequel la machine a été calculée. Cette considération n'intervenant pas dans les machines axiales, il en résulte une possibilité de réversibilité beaucoup plus complète du fonctionnement en pompe et en turbine;

b) Sur le plan pratique, le fait que tout le circuit hydraulique des turbines est convergent, sauf' dans l'aspiration, et au contraire divergent dans les pompes, sauf à l'aspiration.

Si l'on tient compte que la convergence, contrairement à la divergence, tend à s'opposer au décollement, on tire des deux remarques cidessus la conclusion que les soucis des uns sont ignorés des autres, tout au moins dans ce que ces soucis peuvent avoir d'impératif pour réaliser une machine correcte. D'où sans doute Ia réaction habituellement observée, où chacun considère comme inutilisables les expériences de l'autre. 
Cette réaction spontanée s'excuse d'autant mieux lorsqu'on constate que la pratique a conduit à adopter des différences considérables entre les proportions et les tracés des turbines et des pompes de même vitesse spécifique.

Pour ne considérer que les roues, ces différences et leur application sont les suivantes:

Le grand diametre est plus grand dans tous les cas et le petit diamètre plus faible pour les pompes que pour les turbines, surtout pour les petits et moyens $\mathrm{N}_{3}$.

En effet, la pompe exige des canaux relativement longs pour permettre une transmission progressive de l'énergie, avec gradient d'énergie faible, d'abord à l'willard pour éviter la cavitation et ensuite le long de l'aube pour éviter les décollements. Pour la stabilité de l'écoulement, mo valeur de $\mathrm{D} / d$ minimale est nécessaire aux différents $\mathrm{N}_{\text {. }}$.

Pour les lurbines, au contraire, ou les canaux peuvent ctre très convergents, il est intéressant de diminuer $D$ pour limiter la force centrifuge $\mathrm{U}_{\mathrm{D}}{ }^{2} / 2 g$ qui s'oppose à l'écoulement, et d'autre part d'augmenter $d$, ce qui conduit à augmenter débit et puissance pour une même chute; d'où la recherche d'une valeur $\mathrm{D} / d$ la plus faible possible, tendant vers l'unité.

A ces considérations, il y a lieu d'ajouter celles concernant l'influence du dimensionnement sur l'allure de ces caractéristiques. Pour avoir en pompe des caractéristiques à vitesse constante industriellement acceptables, le $\mathrm{V} u / \mathrm{U}$ maximal admissible est environ de 0,6 .

Pour les turbines, au contraire, une grande valeur de $\mathrm{Vu} / \mathrm{U}$ améliore les conditions de fonctionnement a puissance variable sous chute et à vitesse constante. Il n'y a donc aucune raison de ne pas augmenter cette valeur qui permet en outre de réduire les pertes en réduisant les diamètres.

Les valeurs maximales de $V u / \mathrm{U}$ atteintes en turbines sont de l'ordre de 0,9 , ce qui conduit pour ces valeurs extrêmes à un rapport de diamètre de :

$$
\sqrt{\frac{0,9}{0,6}}=1,22
$$

entre pompe et turbine fonctionnant à même

$$
\mathrm{H}_{i}=\frac{\mathrm{UVu}}{g}=\frac{\mathrm{U}^{2}}{g}\left[\frac{\mathrm{V} u}{\mathrm{U}}\right]
$$

et même vitesse de rotation.

Les remarques ci-dessus relatives à la forte convergence des canaux dans les roues de turbines et aux valeurs de $\mathrm{D} / d$ très différentes en pompe et turbine, expliquent aussi les écarts considérables entre les profils méridiens qui rendent des machines de même $N_{s}$ tout à fait mé- comnaissables et sans aucun rapport malgré la théorie commune et en principe réversible.

Par contre, si les écarts de forme et de tracés sont considérables entre les deux types de machines, on peut conclure avec évidence de ce qui précède que si en général une turbine ne peut pas fonctionner en pompe, une pompe au contraire donne presque toujours un fonctionnement en turbine stable, au même titre d'ailleurs qu'un bon divergent fait toujours un bon convergent, et non l'inverse.

Cette simple remarque n'a pas été admise à l'origine sans discussion par les constructeurs de turbines, bien que pendant cette période préalable les spécialistes en pompes la mettaient en cvidence au cours des études expérimentales approfondies quils étaient obligés de faire sur les régimes transitoires fonctionnement en pompe inversée, en turbine centrifuge, en turbine ceniripète...).

En effet, la comnaissance de ces fonctionnements était nécessaire pour résoudre les problemes très particuliers qui se posent à l'arrêt des pompes et qui sont inconnus dans les installalions classiques des turbines, où l'écoulement est gravitaire.

Ces travaux sont intervenus à un moment où les applications des deux techniques allaient devoir enfin se rejoindre par la force des choses pour résoudre par des turbines-pompes réversibles les problèmes d'accumulation, problèmes rendus de plus en plus vastes, de plus en plus complexes par la poursuite sans cesse accrue de nouvelles ressources énergétiques, en particulier pour satisfaire aux consommations de pointes.

Si, jusque-là, spécialistes en turbines et en pompes avaient une tendance à rester chacun avec son mode de penser, n'éprouvant pas la nécessité d'en sortir, si les discussions se placaient sur un plan plus académique et spéculatif que pratique, il allait falloir penser simultanément une même machine sous ses deux aspects, pompe et turbine.

Il allait falloir surmonter les disparités qui opposaient les deux techniques. Or, c'était justement l'étude des causes de ces disparités qui allait permettre de mieux comprendre le domaine de chaque technique, d'en pousser plus loin les limites, et ceci en confrontant les expériences réciproques.

En effet, cette confrontation des difficultés rencontrées, qui sont opposées comme déjà dit pour les pompes et pour les turbines, a permis de préciser plus complètement le rôle joué par les différents facteurs qui, lorsqu'ils étaient favorables, avaient été ignorés ou négligés soit par l'un, soit par l'autre. C'est ainsi qu'a été mieux mise en évidence l'influence du rapport $\mathrm{D} / d$, du 
nombre d'aubes des rones, des diffuseurs ou distributeurs, des profils méridiens dans les fonctionnements au régime optimal et aux régimes transitoires.

Il était naturel qu'on aboutisse à des proportions géométriques intermédiaires, mais cependant plus proches de celles des pompes. Il ne faut pas voir là uniquement un simple compromis, mais bien aussi une extension des possibilités de chacune des techniques qui permet leur recouvrement non seulement sans qu'aucune d'el. les n'ait pratiquement à y perdre, mais en apportant des possibilités nouvelles dans chacum des deux domaines.

Tout ce qui précede porte sur l'hydraulique des machines. Avant d'abandonner cet aspect pour examiner le côté mécanique et technologique de la question, il est nécessaire de signaler l'intérêt que pourait certainement présenter la confrontation du comportement des deux types de machines en face du problème de la cavitation.

A notre connaissance et jusqu'ici une étude systématique n'a pas encore été entreprise, mais elle venait justement d'ètre inscrite au programme de la * Commission de Cavitation de la S.H.F. » lorsque l'activité de cette Commission a été momentanément suspendue. Nous comptons la reprendre incessamment en nous axant sur ce thème, les résultats obtenus par l'observation des turbines seules n'ayant pas permis d'aboutir jusqu'ici à des conclusions directement utilisables dans la pratique.

Il y a lieu de remarquer que pour les turbines les difficultés d'interprétation sont beaucoup plus importantes que dans les pompes; le phénomène est en génèral mieux localisé; les observations sont simplifiées du fait que le fonctionnement a lieu sur une caractéristique unique. Nous espérons qu'il sera fructueux de reprendre l'examen de la cavitation dans les turbines, après une confrontation avec les résultats en pompes.

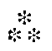

J'arrête un instant cet exposé pour bien préciser que toutes ces considérations ne m'éloignent pas du souvenir de M. Gariel.

La question des machines réversibles devait être une de celles à laquelle il allait être amené par un autre chemin à consacrer tout son intérèt. A l'origine, il y a eu en effet le problème pas. sionnant de la Rance qui a abouti finalement à une des plus belles réussites actuelles. La poursuite de cette réussite est due pour beaucoup à l'esprit créateur et au sens presque prophétique que M. Gariel avait des problèmes nouveaux.

Je parle de réussite au point de vue machine, la réussite du problème de la Rance étant due, comme chacun le sait, à l'acharnement de notre Président, M. Gibrat.
Dans ce problème de la Rance, l'étude des cyeles faite par M. Gibrat a influé progressivement. sur l'évolution des machines qui ont à leur tour influencé le choix du cycle définitif.

Le cadre des machines réversibles à deux fonclionnements a été finalement dépassé pour en arriver à des groupes à quatre fonctionnements, double turbinage et double pompage, ce qui n'est d'ailleurs possible qu'avec des machines axiales à grande vitesse spécifique. Des problèmes complexes des fonctionnements en régimes transitoires se sont aussi posés pour permettre le passage continu d'un fonctionnement à l'autre. En un mot, tous les problèmes relatifs aux turbines et aux pompes se sont trouvés simultanément imbriqués.

Reprenons maintenant notre exposé sur le parallélisme entre pompe et turbine en examinant l'aspect mécanique et technologique.

Dans leur construction, on trouve une parenté presque complète entre machines de mème taille: équilibrage hydraulique des poussées, limitation des pertes par frottements et par fuites, etc... Pour les machines reversibles, le comportement dans le fonctionnement en pompe des directrices mobiles, nécessitées par lé réglage en turbine, pose cependant un problème auquel on doit être attentif; car les directrices sont attaquées par le bord de fuite, ce qui n'est pas sans présenter quelques difficultés.

Si on en vient finalement aux problèmes purement technologiques, en particulier à ceux liés à la nature des produits véhiculés, il $\mathrm{y}$ a lieu de rappeler d'abord que les pompes, par leur universalité, ont dû être adaptées à tous les cas imaginables, alors que, jusqu’à ces dernières années, le seul problème important qui s'était présenté pour les turbines était celui de l'abrasion due au sable, mais toujours avec des concentrations faibles.

Aujourd'hui, par suite du développement général de la technique, les turbines se trouvent déjà et vont se trouver progressivement devant la nécessité de véhiculer des produits de plus en plus variés. Il sera alors salutaire de tenir compte des expériences déjà faites sur les pompes.

Dans ce domaine également, toujours sous l'impulsion de M. Gariel, Neyrpic s'est mis à l'avantgarde de ces problèmes, notamment en poursuivant le développement des turbines à eau de mer avec la Rance et des turbines à boues avec les turbo-foreuses.

Ce dernier cas est aussi à l'avant-garde sur le plan hydraulique puisqu'il s'agit de turbines multicellulaires à plusieurs étages. Or, pour les turbines multicellulaires, on peut dès maintenant leur prédire un avenir beaucoup plus vaste, car pour les machines réversibles à grande hauteur, 
il faudra bien adopter cette solution, la turbine Pelton n'étant elle et malheureusement pas réversible. Là encore la technique des turbines va pouvoir utiliser les expériences acquises dans la technique des pompes.

Que ce soit sur le plan théorique, mécanique ou technologique, on voit que, dans tous les domaines, les techniques des turbines et des pompes sont imbriquées de plus en plus intimement jusqu'à ne former qu'une seule technique: celle des turbo-machines hydrauliques.

Il ne faudrait jamais l'oublier en particulier au cours des discussions entre techniciens, et des rencontres entre spécialistes.

Personnellement, nous regrettons par exemple qu'aux journées d'Aix-en-Provence on ait trop séparé pompes et turbines alor's que certaines questions auraient dû être communes, mais surlout qu'au Congrès de l'A.I.R.H. à Nice le thème n'ait porté que sur les turbines, alors qu'à part la question des aspirateurs de turbines, les autres questions les plus débattues ont été : séparation des pertes, cavitation, et surtout les régimes tran. sitoires se rapportant aussi bien aux pompes qu'aux turbines.

\section{* *}

C'est cette conception d'une technique unique des turbines-machines hydrauliques qui avait déjà prévalu lors des échanges de vues entre Louis Bergeron et Maurice Gariel.

L'attitude de M. Gariel et de ses collaborateurs intimes, dont M. Dagallier en particulier, que nous sommes heureux de voir présider cette séance, a prouvé qu'ils croyaient tous à la fécondité de cette idée et à l'intérêt de la confrontation des expériences réciproques.

Il en est résulté que la collaboration qui s'était établie à l'origine entre M. Gariel et L. Bergeron, s'est poursuivie par la suite avec moi et entre nos équipes.

Cette union a cté couronnée par des réussites communes que nous sommes profondément heureux d'avoir pu partager de son vivant avec celui que nous regrettons tous aujourd'hui.

S'il n'est plus, par contre son esprit demeure en ceux qui l'ont assisté journellement et qui ont partagé sa vie, ainsi que dans les équipes qu'il a formées, comme l'esprit de mon Père demeure en nous. Si la mort a réuni ces deux grandes figures, par contre, sous l'impulsion qu'ils ont su donner, la vie permet à ceux qui restent de continuer en commun l'œuvre qu'ils avaient entreprise, cuvre dans laquelle ils resteront pour nous toujours présents, toujours prêts à encourager nos efforts et à nous montrer la route à suivre.

\section{NOTRE FRONTISPICE}

\section{Cf. page 112}

MONGE Gaspard, conte de Péluse (1746-1818).

Un trait saillant du personnage de Monge est une singulière précocité de l'esprit : a quator\%e ans, il conçoit et exécute de ses ville natale, il construit les instruments d'arpentare plan de sa la même année, il enseigne la physique au célébre collège de l'Oratoire, ì Lyon.

Les dons exceptionnels que cela dénote laissent prévoir la prestigiense carrière que va parcourir ce jeune savant. Né à Beaune (Cotedror) le 9 mai $17+6$, devenu élève de l'école du génie militaire a Mézeres, if $\mathrm{y}$ invente in gémetrie descriptive, puis, it 19 ans, $y$ enseigne les mathematiques. En 1780, il occupe la chaire d'hydraulique fondée au Louvre par Turgot. Ministre de la Marine et des Colonies en 1792, puis organisateur, avec Lavoisier, des salpétrières et des poudreries, Monre professe l'Ecole Normale et devient le principal fondateur de l'Ecole Polytechnique et l'un des 48 premiers membres de l'Institut de Finice.

En Egypte, en 1798, où il présida l'institut du Caire, il donna, le premier, l'explication du mirage.

I'ceuvre de Monge comprend, ontre la déconverte de la géonétrie descriptive, celle du principe de continuité et celle du sens cache des equations aux différences partielles. On lui doit notam ment des ouvrages sur la statique, sur la geométrie descriptive, sur application de laigebre et de lanalyse à la géométrie; ses donte dont le mode de generation est comn, restent placées, selon

Ta Restanation ne sut pas comprendre l'âme de paladin, fidele généreuse, délicate et désintéressée de ce grand savant... qui, dernieres annés avant de mourir à Paris le 28 juillet 1818 .
MONGE Gaspard, conte de Pćluse (1740-1818). Onu of the saliont features of Monge's character is his singularly precoctons mind. He designed and bnit his owon fro pimp when he has fourteen years old and talo years later, for surveying the plan of his native town, he mate all the necessary survey Colicge in Lyons.

The exceptional gifts thus revaled were an indication of the ancaing career that lay before the young scholar. Bom at Beane in the Cote d'Or region of France on the 9th May 17to, he becane a student at the military engineering school at Mizidres, where he incented descriptive geonetry, and where, at the age of nineteon, he became lectwer in mathematics. In 1780 he held the chat of hydranlics folnded by Turgot at the Louve. He was Mi nister for the Navy and Colonies in 1792 and, with Lavoisier, organised the saltpetre works and ponder mills. He was pro fessor at the scole Nomale and became the principal founder of forty-eight members of the Institut de France. In 1798, whilst presiding over the Catro Institute in Egypt, ho aits the first to correctly explain the mirage.

In addition to the discovery of descriptive nometry, Monge was also responsible for the principle of contimity and the latent meaning of partial difference equations. His man worts cover statics, descriptive geometry and the application of alabbra and andysis to geometry. IIs methet for finding the differential canations of surfaces of hnown mans of aneration are, accord. ing to Arago. among the analytical conceptions that cusure immortality for their author.

The Restoration refused to malerstand the chivalrous, fathful, Ginerous, deticule and disinteristed waltere of this great savant. Ho was particularly meanly treated and the distinguished old manl lived out his remaining years in poverty, divested of al 1818 . 